\title{
EchoGéo
}

40 | 2017

Ressources urbaines (2)

\section{Le quartier de la confection à São Paulo}

\section{Sylvain Souchaud}

\section{(2) OpenEdition}

Journals

\section{Electronic version}

URL: https://journals.openedition.org/echogeo/14950

DOI: 10.4000/echogeo.14950

ISSN: 1963-1197

\section{Publisher}

Pôle de recherche pour l'organisation et la diffusion de l'information géographique (CNRS UMR 8586)

\section{Electronic reference}

Sylvain Souchaud, "Le quartier de la confection à São Paulo", EchoGéo [Online], 40 | 2017, Online since 30 June 2017, connection on 10 August 2021. URL: http://journals.openedition.org/echogeo/14950 ; DOI: https://doi.org/10.4000/echogeo.14950

This text was automatically generated on 10 August 2021

EchoGéo est mis à disposition selon les termes de la licence Creative Commons Attribution - Pas d'Utilisation Commerciale - Pas de Modification 4.0 International (CC BY-NC-ND) 


\title{
Le quartier de la confection à São Paulo
}

\author{
Sylvain Souchaud
}

1 Situé à proximité du rio Tietê, dans les quartiers nord de la région centrale, à cheval sur les districts du Bom Retiro, Brás et Pari, le quartier de la confection s'étend sur environ 1000 hectares. La formation de ce secteur urbain remonte à la toute fin du XIX $\mathrm{X}^{\mathrm{e}}$ siècle et au début $\mathrm{du} \mathrm{XX}^{\mathrm{e}}$ siècle, lorsque São Paulo, modeste ville provinciale d'environ 30000 habitants en 1872, connaît, à partir de la décennie suivante un développement sans précédent, porté par l'industrialisation et une immigration internationale. Le Bom Retiro, le Brás, le Pari surgissent alors comme des quartiers péricentraux, industriels, immigrés et ouvriers. Les activités textiles sont, à cette époque, essentielles à ce développement.

2 La confection s'est peu à peu imposée face au textile, au gré de vagues migratoires successives, internationales et internes, et d'une croissance de la consommation urbaine. Aujourd'hui, le quartier de la confection concentre une part conséquente de l'immigration internationale au Brésil et notamment les flux les plus récents, spécialisés dans la confection. Les migrants sud-coréens arrivés dans les années 1970-1980, ainsi que les migrants boliviens et paraguayens présents depuis une vingtaine d'années, se partagent les activités de ce quartier essentiellement commercial où ils travaillent et habitent. La densité de population y est comprise entre 64 et 80 habitants par hectare, c'est l'une des plus basses de la ville et elle est le signe d'une dévalorisation économique et symbolique qui explique en partie la présence des ateliers. 
Illustration 1 - L'intérieur d'un atelier de confection, rue José Paulino, dans le Bom Retiro

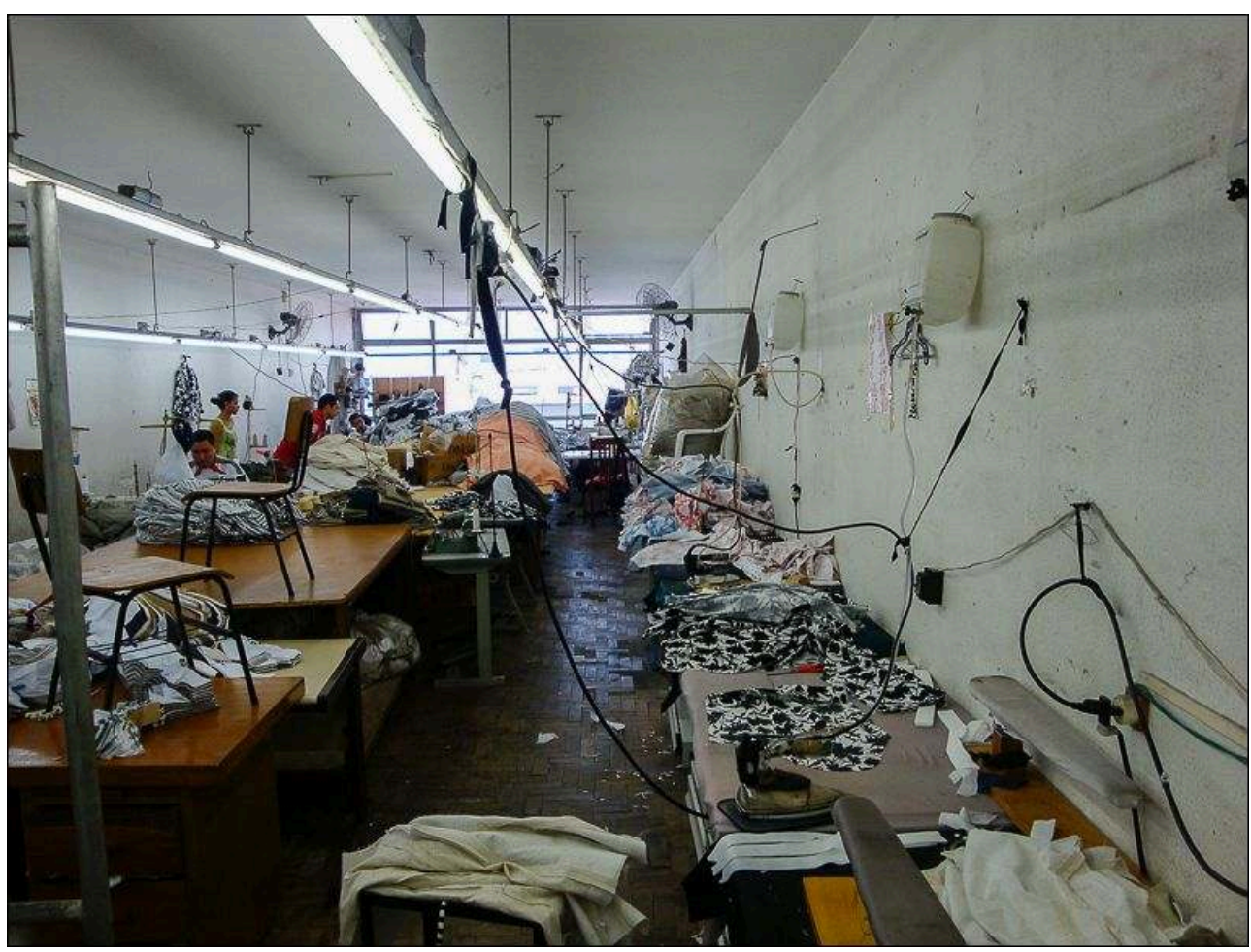

Le patron est coréen, le gérant et la main-d'œuvre sont paraguayens.

Auteur : Sylvain Souchaud, 24 août 2010.

3 La population coréenne (les migrants et une partie de leurs descendants), concentrée dans le Bom Retiro, est plus facilement identifiable que l'immigration bolivienne et paraguayenne, plus diffuse mais non moins nombreuse: les premiers ont investi la création et le commerce vestimentaires, tandis que les seconds qui seraient environ 75000 au total à São Paulo sont spécialisés dans la production.

4 L'activité commerciale commande l'organisation générale du quartier. Elle est répartie dans deux secteurs aux façades d'immeubles colorées et aux vitrines richement décorées qui forment le cœur et la partie la mieux identifiée du quartier. 
Illustration 2 - Le commerce du vêtement dans le Bom Retiro : showrooms de la rua Amorés/Silva Pinto

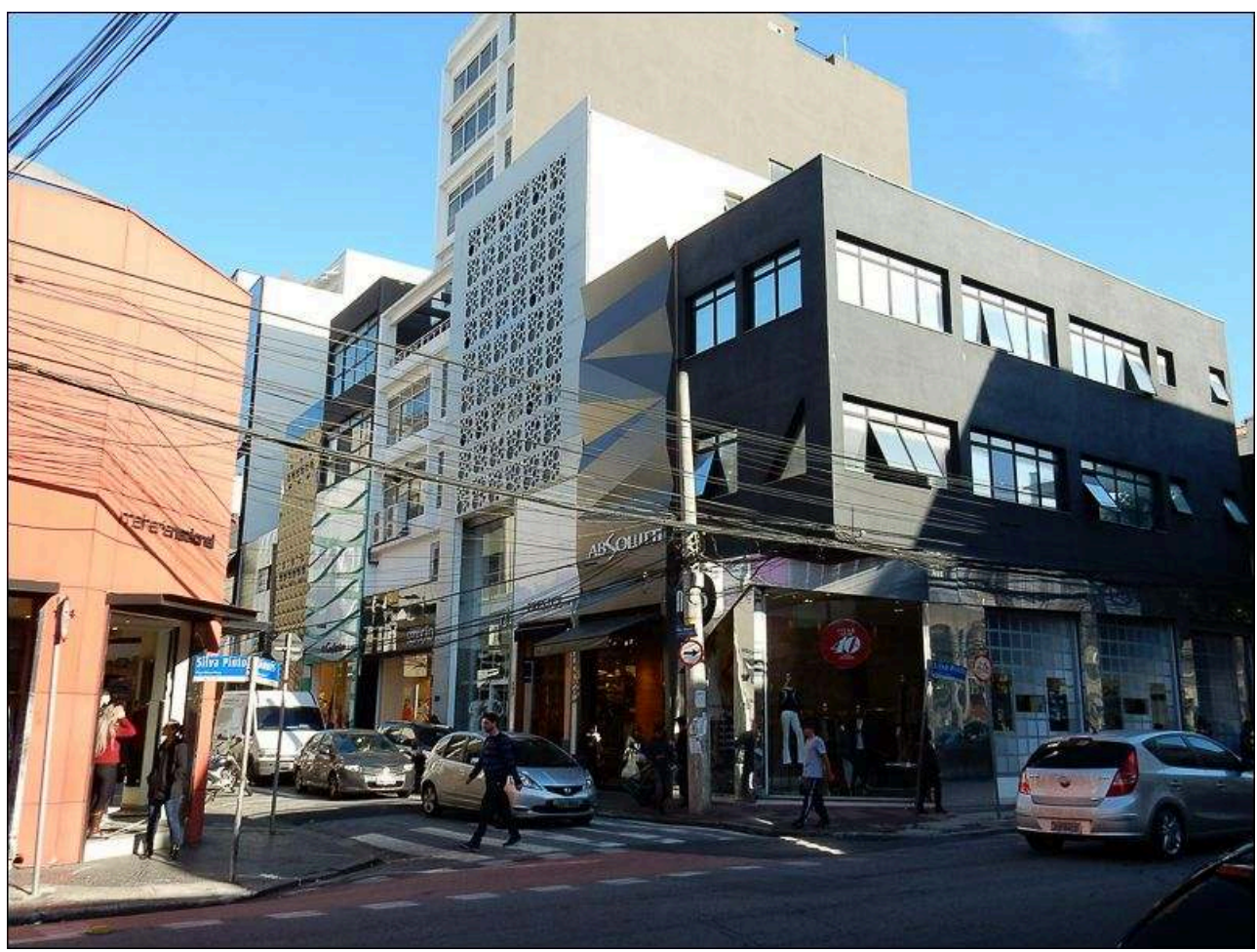

Auteur : Sylvain Souchaud, 14 juin 2016.

5 Ils sont situés dans le Brás et dans le Bom Retiro, à environ deux kilomètres l'un de l'autre. Dans le Bom Retiro, l'offre, plus sophistiquée, est destinée à une classe moyenne ou moyenne supérieure. Dans le Brás, on trouve des vêtements pour les catégories populaires. Le commerce, principalement de gros, est organisé soit dans des magasins sur rue aux vitrines sophistiquées, soit dans de nombreux centres commerciaux spécialisés - surtout dans le Brás) soit, enfin, sur des stands de rue éphémères. 
Illustration 3 - L'arrière d'un centre commercial dédié au vêtement dans le Brás et une aire de stationnement de cars

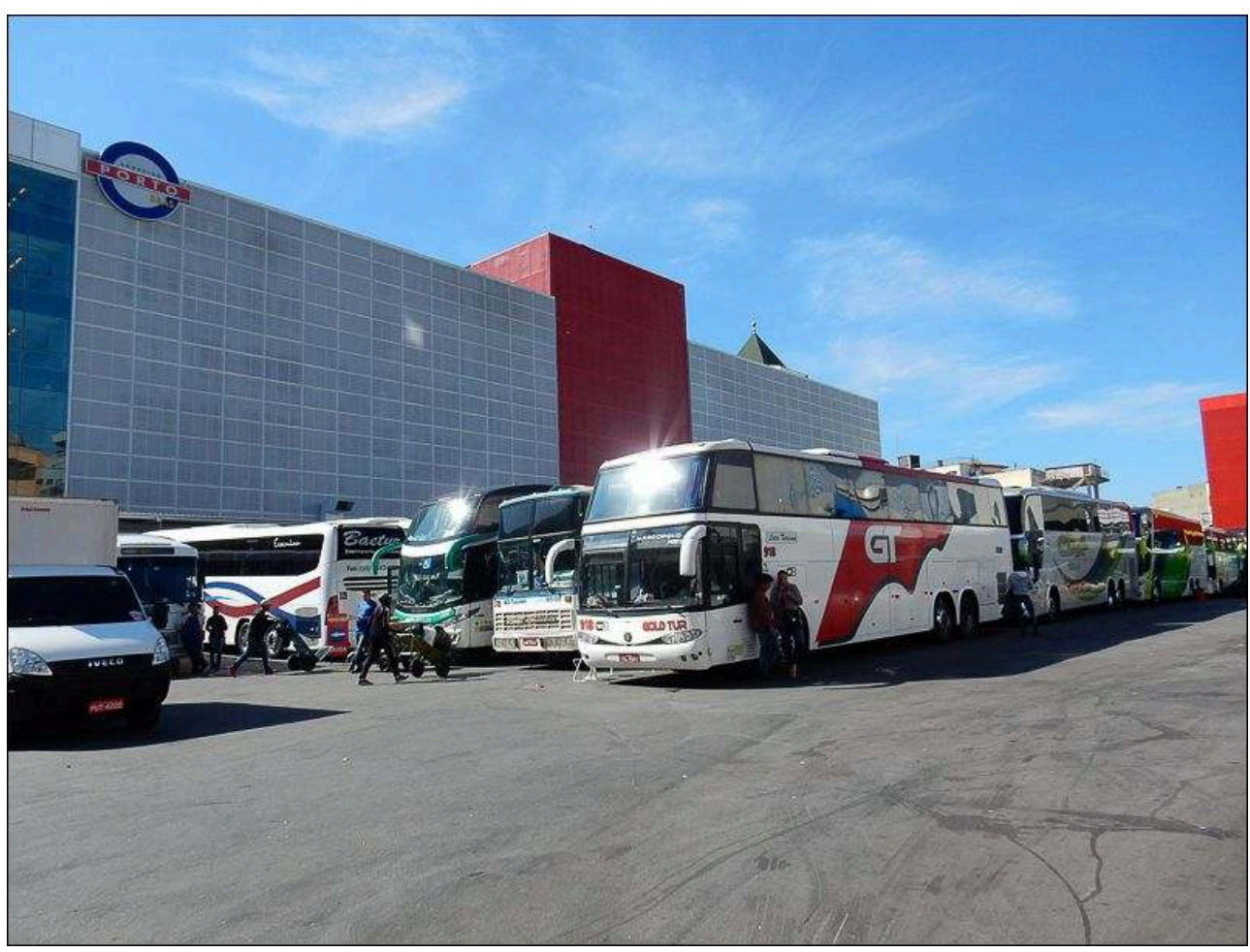

Les cars assurent le transport de touristes nationaux qui viennent s'approvisionner en gros ou au détail dans le quartier.

Auteur : Sylvain Souchaud, 14 juin 2016. 
Illustration 4 - Les boxes d'un centre commercial dans le Brás, Shopping da Juta, rue da Juta

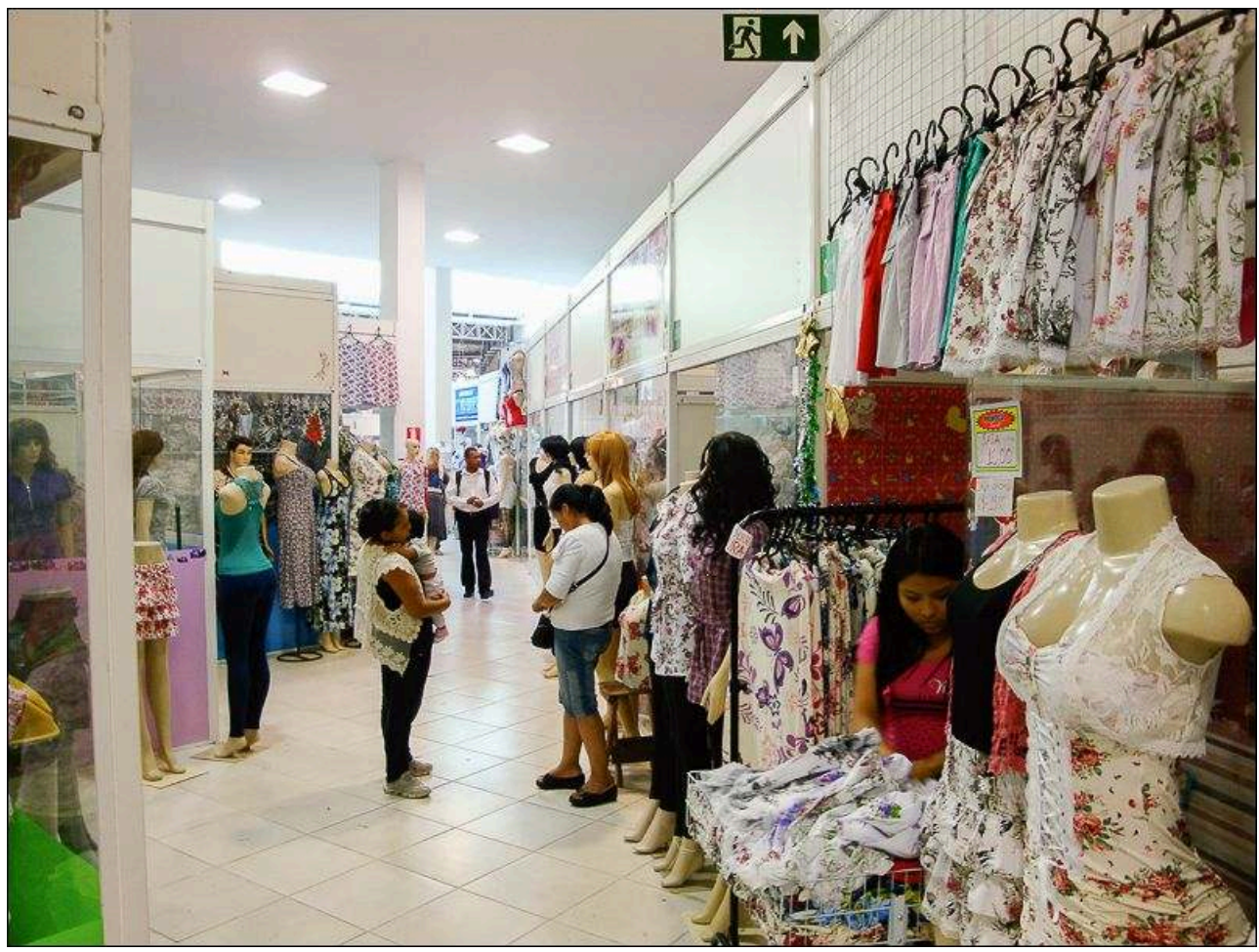

Auteur : Sylvain Souchaud, 16 décembre 2011

Dans les rues adjacentes, la densité de commerces de vêtements diminue et l'on remarque la présence de nombreux fournisseurs (tissus, mercerie, machine à coudre). 
Illustration 5 - Les commerces de tissus dans la rua Mendes Júnior, Brás

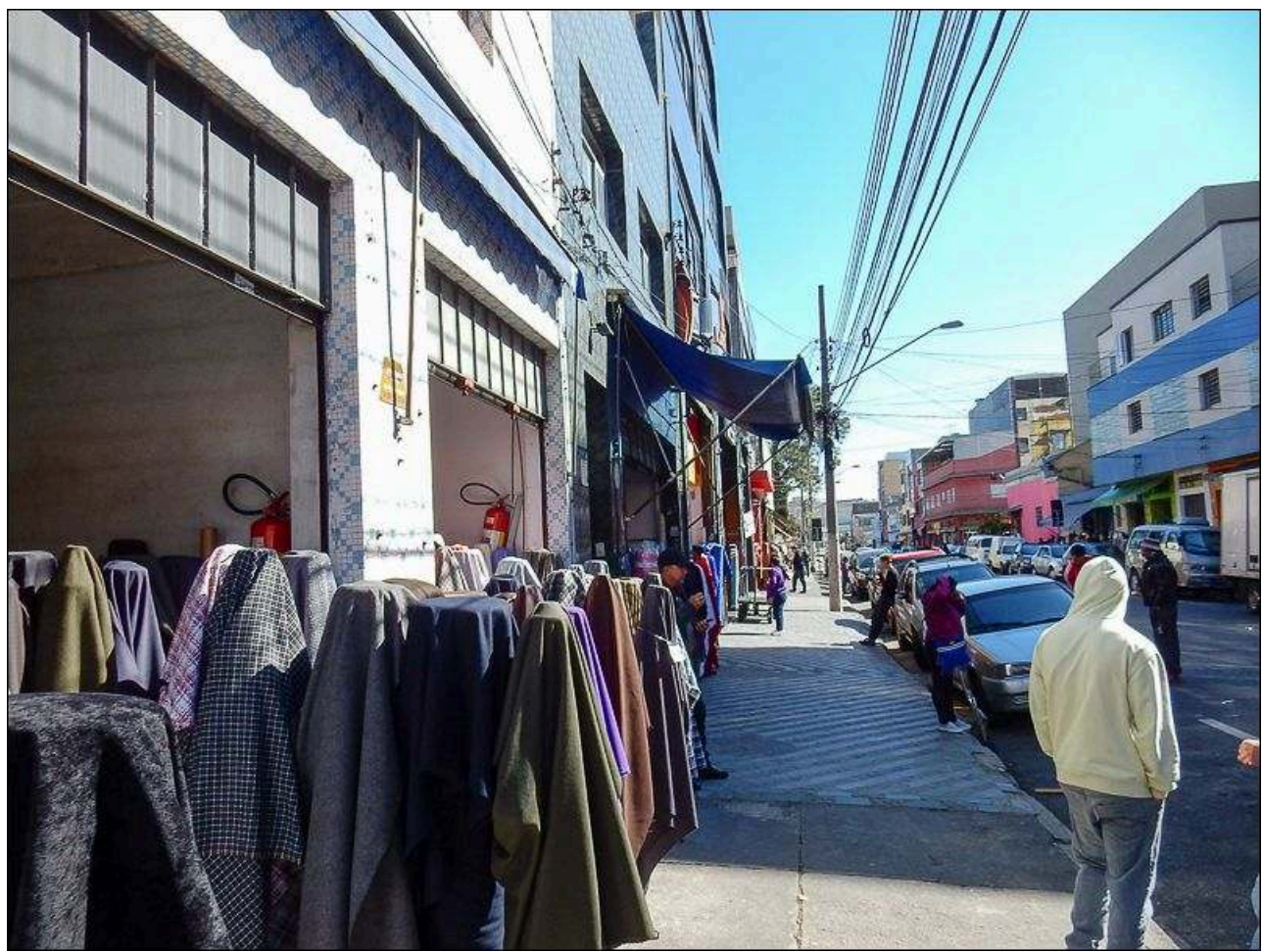

Auteur : Sylvain Souchaud, 14 juin 2016

Illustration 6 - Commerces de machines à coudre et mercerie, rua Newton Prado, Bom Retiro

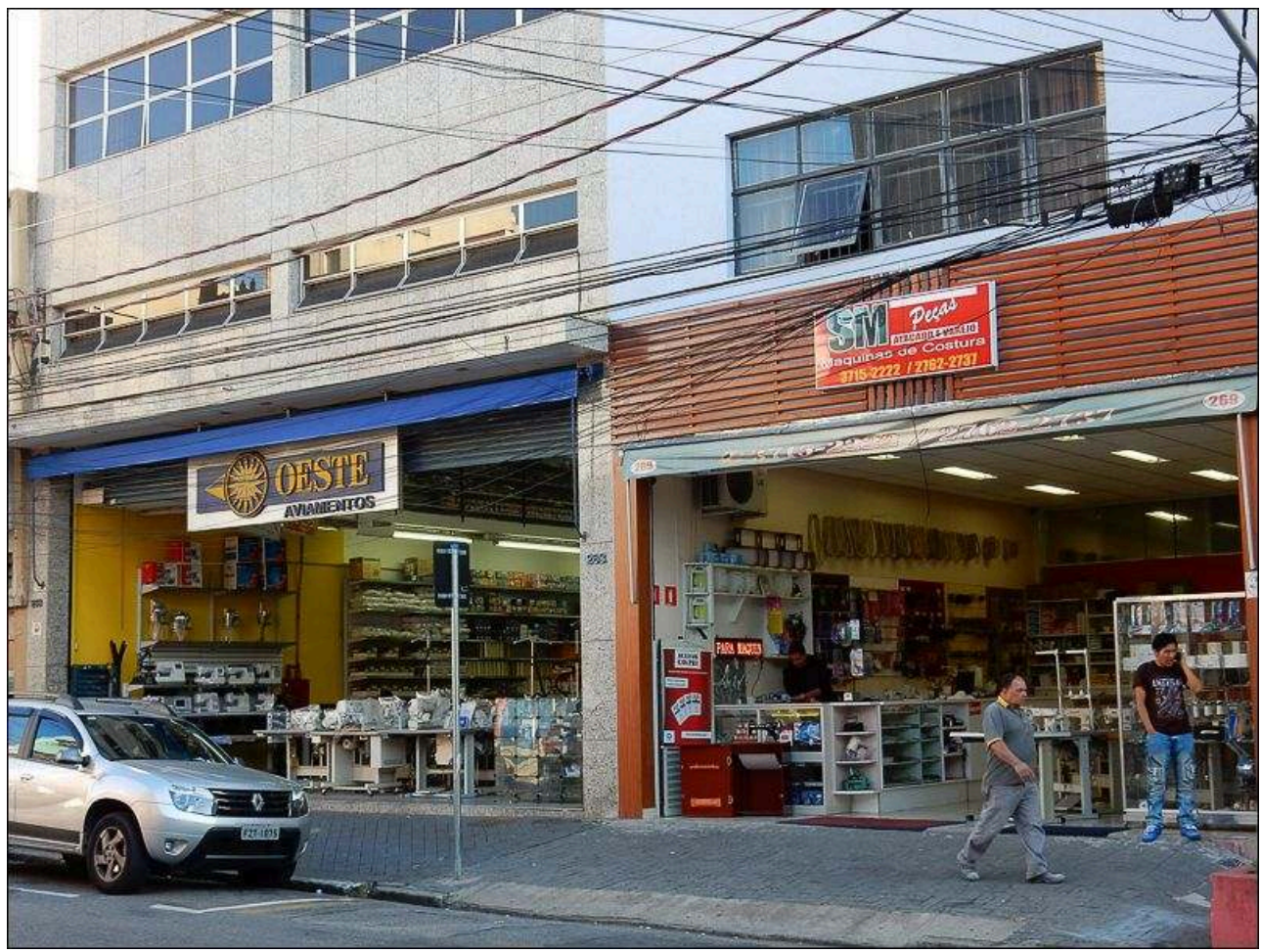

Auteur : Sylvain Souchaud, 14 juin 2016. 
7 Plus loin, dans la couronne limitrophe, résidentielle, alors que l'effervescence est retombée, des activités de soutien (notamment logistiques : stationnements, agences de transport de marchandise et de passagers) et de type commensale (conditionnement et vente de chute de tissus : sulencas) occupent de vastes parcelles.

Illustration 7 - Entrepôt-vente de chutes de tissu reconditionnées (sulancas), rua Almirante Barroso, Brás

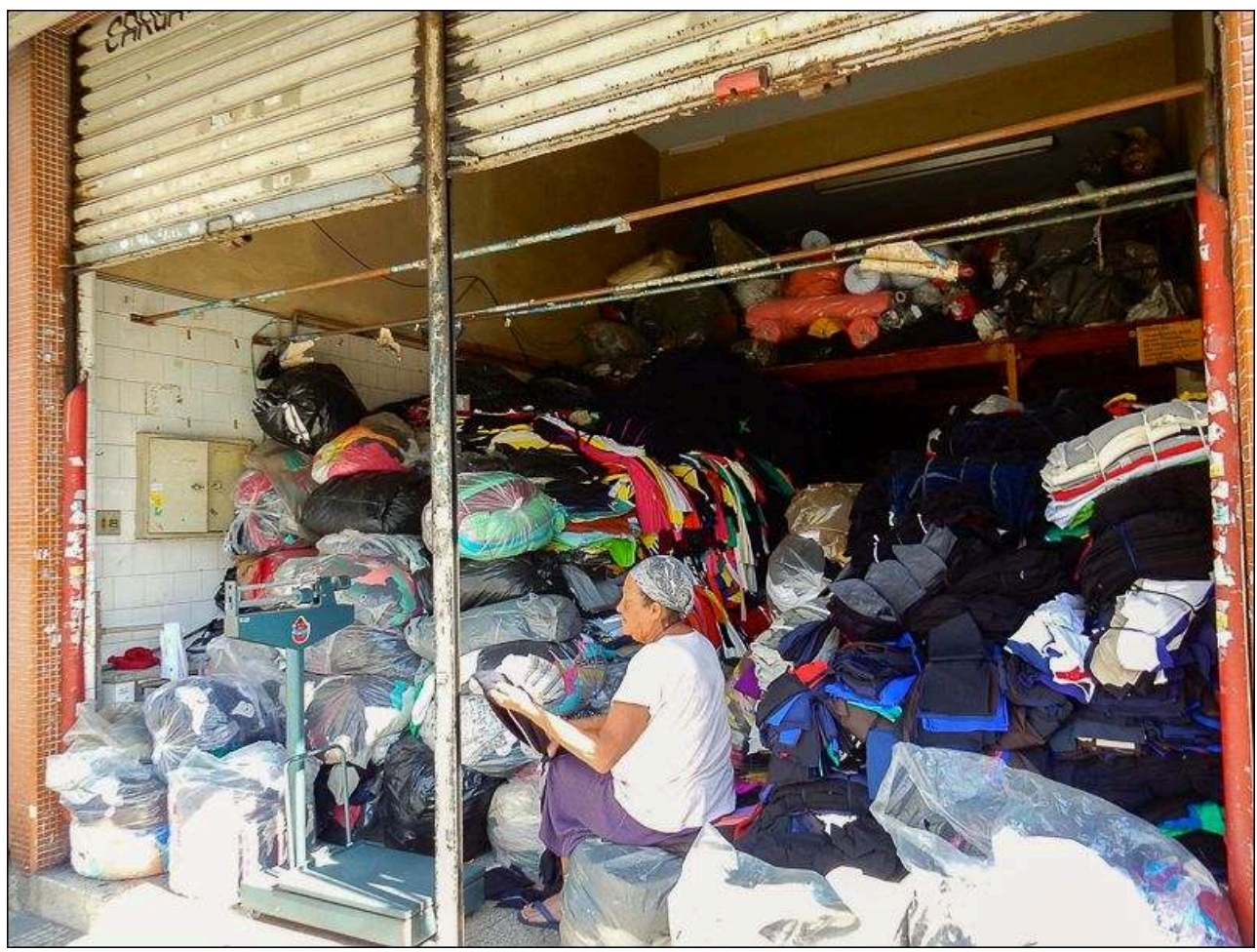

Auteur : Sylvain Souchaud, 14 juin 2016. 
Illustration 8 - Les restes des rouleaux de tissus abandonnés, rua Almirante Barroso, Brás et prêts à être évacués

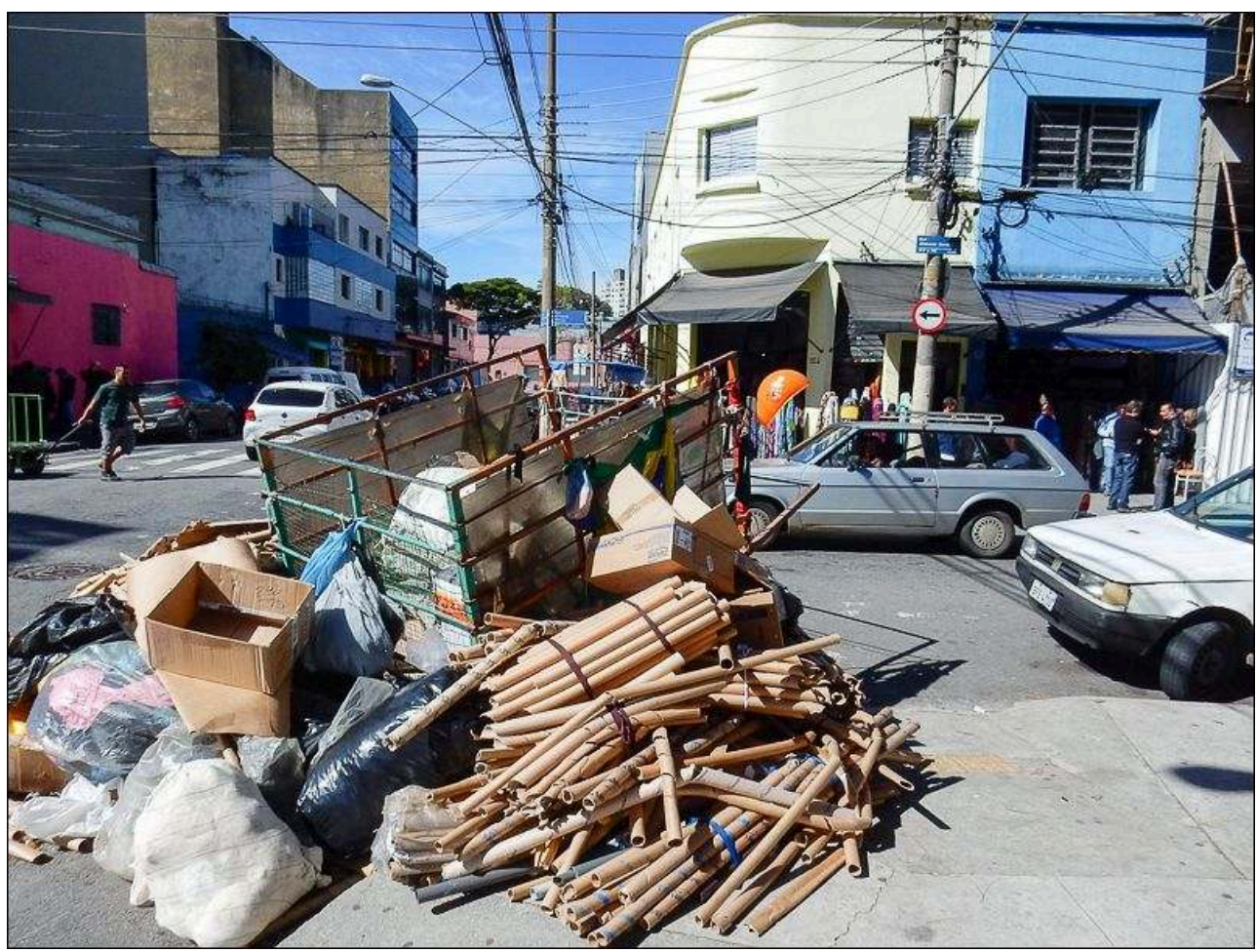

L'évacuation est assurée par une personne (catador) qui collecte les déchets pour recyclage. Auteur : Sylvain Souchaud, le 16 juin 2016.

Cette dernière couronne concentre enfin de nombreuses résidences d'ouvriers, pour l'essentiel des immigrés boliviens ou paraguayens hébergés par leurs employeurs, patrons d'ateliers de confection. Très peu visibles, les ateliers se distribuent sur l'ensemble du quartier, aux étages des bâtiments commerciaux du cœur du quartier, dans les appartements, les arrière-cours et les sous pentes des logements des résidences insalubres. 
Illustration 9 - Intérieur d'une chambre partagée par plusieurs ouvriers paraguayens, mise à disposition par le patron de l'atelier, Bom Retiro

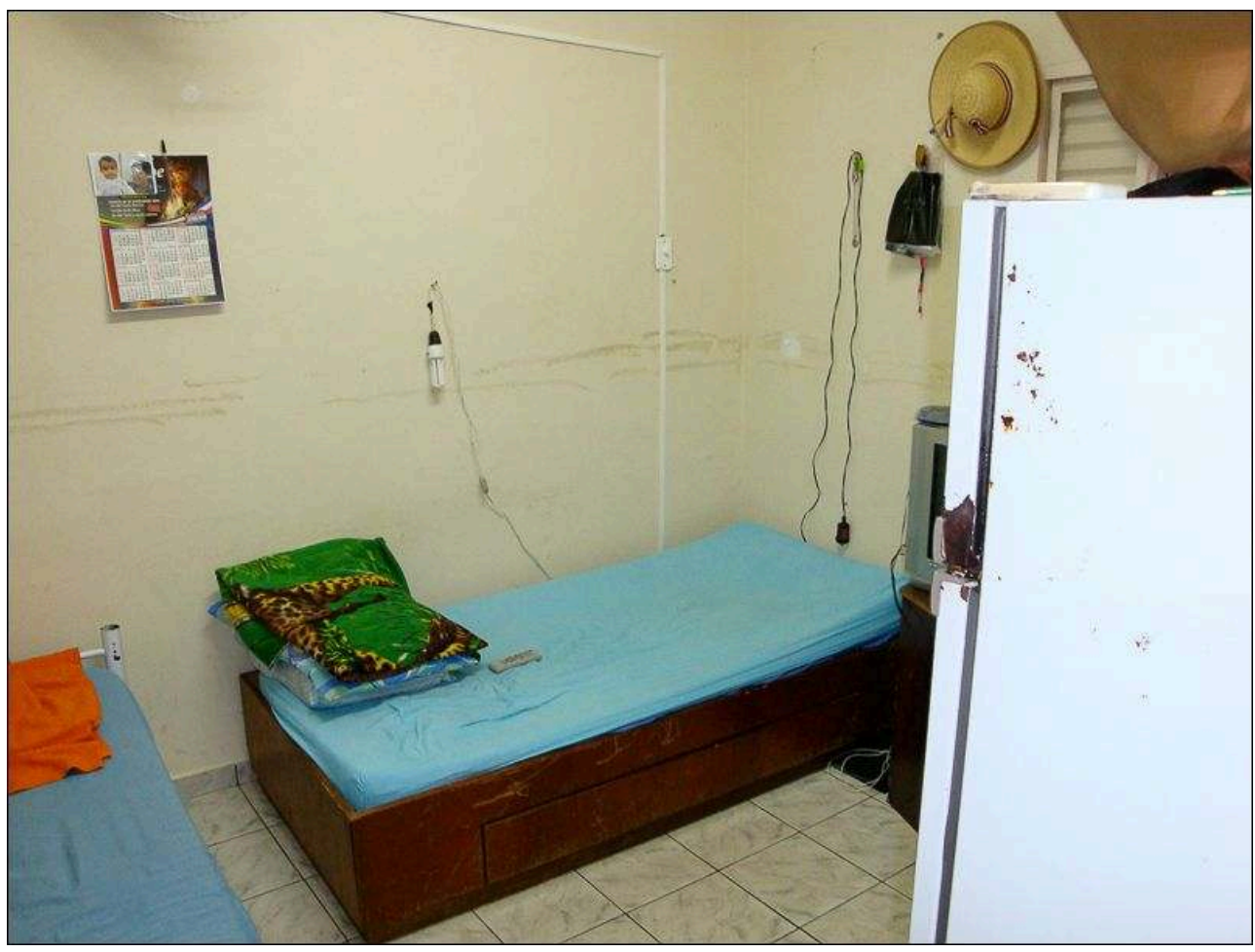

Auteur : Sylvain Souchaud, 25 avril 2010 